\title{
ADEQUATE ULTRAFILTERS OF SPECIAL BOOLEAN ALGEBRAS
}

\author{
BY
}

\section{S. NEGREPONTIS( ${ }^{1}$ )}

ABSTRACT. In his paper Good ideals in fields of sets Keisler proved, with the aid of the generalized continuum hypothesis, the existence of countably incomplete, $\beta^{+}$-good ultrafilters on the field of all subsets of a set of (infinite)

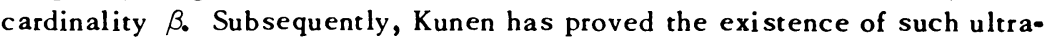
filters, without any special set theoretic assumptions, by making use of the existence of certain families of large oscillation.

In the present paper we succeed in carrying over the original arguments of Keisler to certain fields of sets associated with the homogeneous-universal (and more generally with the special) Boolean algebras. More specifically, we prove the existence of countably incomplete, zood ultrafilters on certain powers of the a-homogeneous-universal Boolean algebras of cardinality $a$ and on the a-completions of the ahomogeneous-universal Boolean algebras of cardinality $a$, where $a=a^{a}>\omega_{b}$ We then develop a method that allows us to deal with the special Boolean algebras of cardinality $a=2 \stackrel{a}{a}$. Thus, we prove the existence of an ultrafilter $p$ (which will be called adequate) on certain powers $\delta \delta_{a}$ of the special Boolean algebra $\delta_{a}$ of cardinality $a$, and the ex= istence of a specializing chain $\left\{\mathcal{C}_{\beta}: \beta<a\right\}$ for $\delta_{\alpha}$, such that $\mathcal{C} \delta_{\beta} \cap p$ is $\beta^{+}$. good and countably incomplete for $\beta<a_{0}$. The corresponding result on the existence of adequate ultrafilters on certain completions of the special Boolean algebras is more technical. These results do not use any part of the generalized continuum hypothesis.

Keisler, in the proof of his fundamental result stated above, made use of a simple set-theoretic lemma on the "disjoint refinement" of certain families of subsets of a given set. In Question 7 of his paper he asks for generalizations of the results of his paper to arbitrary Boolean algebras, considering the special nature of the "disjoint refinement" lemma as a major obstacle to such a generalization. Indeed the results of the present paper rely on the analogue of such a lemma (Lemma 2.1) proved for the Stone space of the homogeneous-universal Boolean

Presented to the Society, May 22, 1970; received by the editors September 19, 1970 and, in revised form, July 5, 1972.

AMS (MOS) subject classifications (1970). Primary 02J05, 02K25, 06A40, 54H10; Secondary 02H13,08A05, 12L10, 54G10.

Key words and phrases. Powers and completions of special, homogeneous-universal Boolean algebras, topological characterization of Stone space of a-homogeneous-universal Boolean algebras, existence of adequate, good, countably incomplete ultrafilters, degree of goodness, weakly anatomic Boolean algebras, generalized continuum hypothesis.

(1) The author acknowledges partial support received from the Canadian National Research Council under grant A-4035. The results of this paper were announced in the Notices Amer. Math. Soc. on May $1970\left[\mathrm{~N}_{3}\right]$. 
algebras. However, the proof of Lemma 2.1 makes use only of one of the two characteristic properties of homogeneous-universal Boolean algebras (described in 1.4(b) below), while (more specifically) the results of Part I use the second characteristic property of such Boolean algebras (given in 1.4(c) below) as well (in proving Lemma 2.2).

The paper is organized in two parts. In Part I we consider powers of homogeneous-universal and special Boolean albegras. In $\$ 2$, Lemma 2.1 on disjoint refinements and Lemma 2.2, which is the analogue of Keisler's Lemma 4C in $\left[\mathrm{K}_{4}\right]$, are established, leading to the proof of Theorem $A$. The main result of Part I on the existence of adequate ultrafilters (Theorem $B$ ) is proved for all special Boolean algebras of cardinality $a=2^{a}>\omega$. Part II deals with certain partial completions of homogeneous-universal and special Boolean algebras. In $\$ 4$, the proof is given on the existence of countably incomplete, $a$-good ultrafilters on the $a$-completion of the $a$-homogeneous-universal Boolean algebras of cardinality $a$ (Theorem $C$ ). An almost trivial, but basic, observation here is that the a-completion $\mathcal{C}^{(a)}$ of an $a$-homogeneous-universal Boolean algebra $\mathcal{C}$ can be identified with the a-complete field of subsets of the Stone space $s(\mathcal{C})$ of $\mathcal{C}$, a-generated by $\mathcal{C}$ (4.2). The existence proof here is simpler than its analogue in Part I, mainly because of any lack of complications in the proof of the analogue of 2.2. Theorem $C$ can be generalized, by replacing in its statement " $a$-homogeneous-universal" by "atomless, weakly a-atomic" (essentially in the sense of Pierce [P]) ( $\$ 4$, Theorem $\left.C^{\prime}\right)$. However, the methods for completions of special Boolean algebras in $\$ 5$ do not work out as well, as for products in $\$ 3$. We are forced to consider a chain of fields of sets that is not as closely connected with a specializing chain for the special Boolean algebras, as one would wish; also we have to give separate arguments for the regular cardinals and for the strong limit cardinals.

The results of both Parts I and II may be considered as particular (indeed, extreme) cases of a general procedure on partial completions, which is outlined in the remarks at the end of $\$ 4$.

The results of $\mathrm{Part}$ II may be used for various model-theoretic applications, analogous to those given by Keisler $\left[K_{1}\right],\left[K_{2}\right],\left[K_{3}\right],\left[K_{5}\right],\left[K_{6}\right],\left[K_{7}\right]$, Kochen $\left[K_{0}\right]$, Galvin [G], and especially Mansfield $\left[M_{1}\right],\left[M_{2}\right]$, without making any use of the generalized continuum hypothesis. These applications however are rather cumbersome and in view of the definitive work of Shelah [Sh] on the characterization of elementary equivalence in terms of ultrapowers they will not be given. The more satisfactory and in a sense deeper results of Part I do not seem to lend themselves to similar applications.

1. Preliminaries. The axiom of choice is assumed. Ordinal numbers are denoted by $\xi, \zeta, \eta, \lambda$ with or without subscripts. An ordinal coincides with the 
set of all smaller ordinals, i.e. $\xi<\zeta$ is equivalent to $\xi \in \zeta$. Nevertheless, we make the notational distinction between the first ordinal 0 and the empty set $\varnothing$. A cardinal number is an initial ordinal. Cardinals are denoted by $a, \beta, \gamma, \delta$. $0,1, \ldots, n, k, \ldots$ denote natural numbers. The first infinite cardinal is $\omega$. The least cardinal greater than $\beta$ is denoted by $\beta^{+}$. The cardinality of the set of all mappings from $\beta$ to $\alpha$ is denoted by $\alpha^{\beta}$. The generalized continuum hypothesis (G.C.H) states that $\alpha^{+}=2^{a}$ whenever $\omega \leq \alpha$. The cardinality of a set $A$ is denoted by $|A|$. A set of cardinality $\alpha$ (and no additional structure) will usually be identified with $a$. For a nonempty set $A$, let $S_{a}(A)$ denote the set of all nonempty subsets of $A$ of cardinality less than $a$. For a limit ordinal $\lambda(\lambda \neq 0)$, $\operatorname{cf}(\lambda)$ denotes the cofinality of $\lambda$. A cardinal $\alpha$ is regular if $\alpha=\operatorname{cf}(\alpha)$, singular otherwise. If $\alpha$ is equal to $\beta^{+}$for some cardinal $\beta$, then $\alpha$ is called a nonlimit cardinal, otherwise a limit cardinal. A cardinal $\alpha$, satisfying the condition $2^{\beta}<$ $\alpha$ whenever $\beta>\alpha$, is a strong limit cardinal. If $\alpha$ is a regular and a limit cardinal, then $\alpha$ is inaccessible; if $\alpha$ is a regular and a strong limit cardinal, then $\alpha$ is strongly inaccessible. The beths $\beth_{\xi}$ are defined inductively by $\beth_{0}=\omega$ and $\beth_{\xi}=\Sigma\left\{2 \beth^{\prime}: \zeta<\xi\right\}$ for $\xi \neq 0$. We set $a^{\beta}=\Sigma\left\{\alpha^{\gamma}: \gamma<\beta\right\}$. The following results are well known (cf. the papers of Hausdorff $\left[\mathrm{H}_{1}\right]$ and Tarski $[\mathrm{T}]$ for 1.1(b), the text of Bachmann [B], especially $\S \S 33-34$, and Morley-Vaught [MV] for an explicit statement of 1.1(d)).

1.1. Let $a$ be an infinite cardinal. Then,

(a) $\alpha$ is a strong limit cardinal if and only if $a=J_{\lambda}$ where $\lambda=0$ or $\lambda$ is a limit ordinal;

(b) Let $\lambda$ be a (nonzero) limit ordinal. Then $\operatorname{cf}\left(د_{\lambda}\right)=\operatorname{cf}(\lambda)$;

(c) $a=a^{a}$ if and only if $a=2^{a}$ and $\alpha$ is regular;

(d) $a=2^{a}$ if and only if either $a=a^{a}$ or $a$ is a strong limit cardinal;

(e) [G.C.H.] $\alpha=a^{a}$ if and only if $\alpha$ is regular if and only if either $\alpha$ is a nonlimit cardinal or $\alpha$ is (strongly) inaccessible;

(f) [G.C.H.] $a=2^{a}$ holds for all (infinite) cardinals $a$.

Note that it follows from 1.1(a), (b), and (d) that there are cardinals $a$ of arbitrarily large cofinality, such that $a=2^{a}$. On the other hand, no uncountable cardinals $a$ such that $a=\alpha^{a}$ are known to exist without some additional assumptions, such as the generalized continuum hypothesis, or the existence of strongly inaccessible cardinals.

The general theory of Jónsson classes has been developed in $\left[\mathbf{J}_{\mathbf{l}}\right],\left[\mathbf{J}_{2}\right],\left[\mathbf{K}_{2}\right]$, [CK]. A convenient reference is the text of Bell-Slomson [BS]. We need here the results of the general theory, only as they apply to the Jónsson class of Boolean algebras.( ${ }^{2}$ ) A Boolean algebra $\mathcal{C}$ is $a$-homogeneous if for any Boolean algebra $\mathfrak{I}$ of cardinality less than $a$, and any embeddings $b_{1}, b_{2}: \mathfrak{I} \rightarrow \mathcal{C}$, there is an

(2) Alternatively, we may consider the complete and model-complete theory of atomless Boolean algebras (cf. Lemma 3 in [Jo]). 
automorphism $b$ of $\mathcal{C}$, such that $b \circ b_{1}=b_{2}$. A Boolean algebra $\mathcal{C}$ is a-universal if given any Boolean algebra $\mathfrak{I}$ of cardinality at most $\alpha$, there is an embedding $b: \mathfrak{D} \rightarrow \mathcal{C}$. If $\mathcal{C}$ is $a$-homogeneous and a-universal, then $\mathcal{C}$ is called $a$-homogeneousuniversal. For the notion of special, we adopt the following simplified definition (equivalent to the original definition by Morley-Vaught [MV]), given by Chang-Keisler [CK]. Let $a$ be an (infinite) cardinal. A Boolean algebra $\mathcal{C}$ is called special of cardinality $\alpha$ if $|\mathcal{C}|=\alpha$ and if $\mathcal{C}$ has a specializing chain, i.e. $\mathcal{C}$ is equal to the union of a chain $\left\{\mathcal{C}_{\beta}: \beta<\alpha\right\}$ of Boolean subalgebras of $\mathcal{C}_{\text {, whe }} \mathcal{C}_{\beta}$ is $\beta^{+}$-homogeneous-universal for all cardinals $\beta$ less than $\alpha$. We need the following facts.

1.2. Let $\beta$ be any infinite cardinal and let $\mathcal{C}_{0}$ be any Boolean algebra of cardinality at most $2^{\beta}$. Then, there is a $\beta^{+}$-homogeneous-universal Boolean algebra $\mathcal{C}$ of cardinality $2^{\beta}$, and a Boolean algebra embedding of $\mathcal{C}_{0}$ into $\mathcal{C}$. (This statement is a particular case of a result by Morley-Vaught; cf. Theorem 2.10 and Remark (4) following Theorem 2.8 in [MV]).

1.3. Let $a=2^{a}$. There is up to isomorphism exactly one special Boolean algebra of cardinality $a$. (This Boolean algebra will be denoted by $\mathcal{S}_{\alpha^{*}}$ ) Furthermore, if $\alpha$ is regular, then $\mathcal{S}_{a}$ is the unique, up to isomorphism, $a$-homogeneousuniversal Boolean algebra of cardinality $a$. (This statement is a particular case of Theorem 2.8 in [MV].)

We make use of Stone's duality between Boolean algebras and compact zerodimensional spaces. The Stone space of a Boolean algebra $\mathcal{C}$ is denoted by $S(\mathcal{C})$. We identify $\mathcal{C}$ with the field of open-and-closed subsets of $S(\mathcal{C})$, or with a field of sets of an appropriate subspace of $S(\mathcal{C})$. Let $S$ be a zero-dimensional space and $U$ an open subspace of $S$. The type $r(U)$ of $U$ is the least cardinal $a$, such that $U$ is equal to the union of a open-and-closed subsets of $S$. We need the following facts:

1.4. Let $\mathcal{C}$ be an (infinite) $a$-homogeneous-universal Boolean algebra. Then $S(\mathcal{C})$ satisfies the following conditions:

(a) $S(\mathcal{C})$ has no isolated elements;

(b) if $U$ is open and $1<\tau(U)<\alpha$, then $U$ is not dense in $S(\mathcal{C})$;

(c) if $U$ and $V$ are two disjoint open sets, such that $r(U)+\tau(V)<\alpha$, then there is an open-and-closed set $W$, such that $U \subset W$ and $V \cap W=\varnothing$.

(These statements are given in Corollary 1.5 and Theorem 1.7 in $\left[\mathrm{N}_{1}\right]$; they are stated there only for the $a$-homogeneous-universal Boolean algebra of cardinality $a$, but the additional assumption is not needed for their proof.)

Let $\mathcal{F}$ be a field of sets. An ultrafilter $p$ on $\mathfrak{F}$ is countably incomplete if there is a sequence $\left\{Z_{n}: n<\omega\right\}$ of elements of $p$ such that $\bigcap_{n<\omega} Z_{n}=\varnothing$. We introduce the notion of a good ultrafilter on a field of sets in analogy to Keisler's original definition in $\left[K_{4}\right]$. A map $\phi: S_{a}(\beta) \rightarrow \mathcal{F}$ is monotone if $\phi(F) \subset \phi\left(F^{\prime}\right)$ 
for $F, F^{\prime} \in S_{\omega}(\beta)$, such that $F \supset F^{\prime}$, and multiplicative if $\phi\left(F \cup F^{\prime}\right)=\phi(F) \cap$ $\phi\left(F^{\prime}\right)$ for $F, F^{\prime} \in S_{\omega}(\beta)$. An ultrafilter $p$ on $\mathcal{F}$ is $\alpha$-good if for every $\beta<\alpha$ and every monotone mapping $\phi: S_{\omega}(\beta) \rightarrow p$, there is a multiplicative mapping $\psi$ : $S_{\omega}(\beta) \rightarrow p$, such that $\psi(F) \subset \phi(F)$ for all $F \in S_{\omega}(\beta)$ (denoted by $\psi \leq \phi$ ). We set $F^{*}=\mathfrak{F} \backslash\{\varnothing\}$. If $E \subset F^{*}$ and $\alpha$ is an infinite cardinal, we let $\operatorname{Mon}(\alpha, E)$ denote the set of all monotone mappings $\phi: S_{\omega}(\beta) \rightarrow E$ for all $\beta<\alpha$, and Mult $(\alpha, E)$ the set of all multiplicative mappings $\phi: S_{\omega}(\beta) \rightarrow E$ for all $\beta<\alpha$. Let further $b$ : $\mathcal{F} \rightarrow \mathcal{G}$ be an embedding of fields of sets. There are obvious induced embeddings, denoted by

$$
\operatorname{Mon}(a, b): \operatorname{Mon}(a, E) \rightarrow \operatorname{Mon}(\alpha, b[E]),
$$

etc. An ultrafilter $p$ on $\varrho$ is called $(\alpha, E)-\operatorname{good}$ if $b[E] \subset p$ and for every $\phi \epsilon$ $\operatorname{Mon}(\alpha, E)$, there is $\psi \in \operatorname{Mult}(\alpha, p)$ such that $\psi \leq \operatorname{Mon}(\alpha, b) \phi$. We will not give a general definition of an adequate ultrafilter, but refer to Theorems B and D for its meaning in each case.

Let $\mathcal{C}$ be a Boolean algebra and let $\delta$ be an infinite cardinal. We identify, via Stone's duality, the power $\mathcal{C}^{\delta}$ with the field of all open-and-closed subsets of $S(\mathcal{C}) \times \delta$, the set $\delta$ being given the discrete topology. For a zero-dimensional space $S$, we let $\mathfrak{B}(S)$ denote the Boolean algebra of all open-and-closed subsets of $S$. Thus, $\mathcal{C}^{\delta}$ and $\mathfrak{B}(S(\mathcal{C}) \times \delta)$ are isomorphic. An ultrafilter $p$ on $\mathcal{C}^{\delta}$ will be called $\delta$-uniform if for every $e \in p$, the set

$$
\{\lambda<\delta:(S(\mathcal{C}) \times\{\lambda\}) \cap e \neq \varnothing\}
$$

has cardinality $\delta$. Let $b: \mathcal{C} \rightarrow \mathcal{C}_{1}$ be a Boolean algebra embedding. The induced continuous epimorphism of Stone spaces is denoted by $S(b): S\left(\mathcal{C}_{1}\right) \rightarrow s(\mathcal{C})$, and we let $S(b) \times \mathrm{id}_{\delta}: S\left(\mathcal{C}_{1}\right) \times \delta \rightarrow S(\mathcal{C}) \times \delta$ be given by $\left(s(b) \times \mathrm{id}_{\delta}\right)=(S(b) s, \lambda)$. Some notation is also used in $\$ 3$ concerning direct systems and direct limits, information on which can be found in Chapter VIII of the text of Eilenberg-Steenrod [ES].

Let $\alpha$ be an infinite cardinal. A Boolean algebra $\mathcal{C}$ is $a$-complete if every subfamily of $\mathcal{C}$ of cardinality less than $\alpha$ has a supremum in $C$. A field of sets $\mathcal{F}$ is $\alpha$-complete if for every $\beta<\alpha$ and every family $\left(A_{\xi}\right)_{\xi<\beta} \subset \mathcal{F}$, the set-theoretic union $\bigcap_{\xi<\beta} A_{\xi}$ is an element of $\mathcal{F}$. We note that in general an $\alpha$-complete Boolean algebra is not isomorphic to an $\alpha$-complete field of sets. A subset $D$ of a Boolean algebra $\mathcal{C}$ is dense (in $\mathcal{C}$ ) if, for every $c \in \mathcal{C}, c \neq \varnothing$, there is $d \in D$, such that $\varnothing \neq d \subset c$. Let $S$ be a subset of an $a$-complete Boolean algebra $\mathcal{C}$. The intersection of all $a$-complete Boolean subalgebras of $\mathcal{C}$ containing $S$ is called the a-complete Boolean subalgebra of $\mathcal{C}$ a-generated by $S$. The following fact is easily established.

1.5. Let $\alpha$ be a regular cardinal and let $\mathcal{C}$ be an $\alpha$-complete Boolean algebra 
a-generated by a subset of cardinality at most $\beta$. Then $|\mathcal{C}| \leq \beta^{a}$.

Let $b: \mathcal{C} \rightarrow \mathfrak{D}$ be a Boolean algebra embedding. The pair $\langle\mathscr{D}, b\rangle$ is called an a-completion of $\mathcal{C}$ if $b[\mathcal{C}]$ is a dense subalgebra of $\mathscr{D}, \mathfrak{D}$ is a-complete, and $\mathfrak{D}$ is a.generated by $b[\mathcal{C}]$.

1.6. Let $a$ be an infinite cardinal and let $\mathcal{C}$ be a Boolean algebra. Then, there is an $a$-completion $\langle\mathscr{D}, b\rangle$ of $\mathcal{C}$, which is unique in the following sense: if $\left\langle\mathfrak{D}^{\prime}, b^{\prime}\right\rangle$ is an a-completion of $\mathcal{C}$, then there is an isomorphism $g$ of $\mathfrak{D}$ onto $D^{\prime}$ such that $g \circ b=b^{\prime}$.

We will consider $\mathcal{C}$ as a subalgebra of its unique $a$-completion, which will be denoted by $\mathcal{C}^{(a)}$.

The reader is referred to the text of Sikorski [S] for detailed information on Boolean algebras. (Note, however, that Sikorski's notion of $\alpha$-completeness coincides with our notion of $\alpha^{+}$-completeness.)

\section{PART I: PRODUCTS}

2. Good ultrafilters on products of homogeneous-universal Boolean algebras. In the present section we prove two lemmas (the first of which will be of use for the remainder of the paper), and Theorem A on the existence of countably incomplete, good ultrafilters on certain powers of the homogeneous-universal Boolean algebras. The first lemma (on "disjoint refinements") is the analogue of Lemma $4 \mathrm{~A}$ of $\left[\mathrm{K}_{4}\right] \cdot(3)$

2.1. Lemma.(4) Let $\mathcal{C}$ be an a-bomogeneous-universal algebra, let $\beta<\alpha$, and let $\left(V_{\xi}\right)_{\xi<\beta}$ be a family of nonempty open-and-closed subsets of $S(\mathcal{C})$. Then, there is a family $\left(W_{\xi}\right)_{\xi<\beta}$ of nonempty, open-and-closed subsets of $S(\mathcal{C})$, such that $W_{\xi} \subset V_{\xi}$ for $\xi<\beta$, and $W_{\xi} \cap W_{y}=\varnothing$ for $\xi<\zeta<\beta$.

Proof. We employ transfinite induction on $\beta$. Let $W_{0}^{(0)}=V_{0}$. Let $\xi<\beta$ and assume inductively that we have defined sets $w_{\eta}^{(\zeta)}$ for $\eta \leq \xi$ and $\eta \leq \zeta<\xi$, such that

$$
\begin{aligned}
& V_{0}=W_{0}^{(0)} \supset W_{0}^{(1)} \supset \ldots \supset W_{0}^{(\eta)} \supset \ldots \supset W_{0}^{(\zeta)} \supset \ldots \\
& V_{1} \supset \supset W_{1}^{(1)} \supset \ldots \supset W_{1}^{(\eta)} \supset \ldots \supset W_{1}^{(\zeta)} \supset \ldots \\
& V_{\eta} \supset \quad \supset W_{\eta}^{(\eta)} \supset \ldots \supset W_{\eta}^{(\zeta)} \supset \ldots
\end{aligned}
$$

(3) Lemma $4 \mathrm{~A}$ of $\left[\mathrm{K}_{\mathbf{4}}\right]$ has been obtained earlier as Lemma 1 in Kuratowski [Kur] and as Lemma 4.6 in Erdös-Gillman-Henriksen [EGH].

(4) The proof of this lemma is also given in $\left[\mathrm{N}_{2}\right]$; it is repeated here for completeness. 
where

$$
\begin{aligned}
& w_{\eta}^{(\zeta)} \text { is a nonempty, open-and-closed subset of } s(\mathcal{C}), \\
& W_{\eta}^{(\zeta)} \cap W_{\eta^{\prime}}^{(\zeta)}=\varnothing \text { for all } \eta, \eta^{\prime} \leq \zeta, \eta \neq \eta^{\prime}
\end{aligned}
$$

We define $W_{\eta}^{(\xi)}$ for all $\eta$, such that $\eta \leq \xi$. Note that the set $\bigcap_{\eta \leq \zeta<\xi} W_{\eta}^{(\zeta)}$ is nonempty, by the compactness of $S(\mathcal{C})$, and it has nonempty interior, for every $\eta<\xi$, by $1.4(\mathrm{~b})$. Let $\widetilde{W}_{\eta}^{(\xi)}$ be any nonempty open-and-closed subset of $\bigcap_{\eta \leq \zeta<\xi} W_{\eta}^{(\zeta)}$. Clearly the family $\left\{\widetilde{W}_{\eta}^{(\xi)}\right.$ : all $\eta$, such that $\left.\eta<\xi\right\}$ consists of pairwise disjoint, nonempty subsets of $S(\mathcal{C})$.

We now introduce the set $V_{\xi}$, and employ a subsidiary transfinite induction on all ordinals $\eta, \eta<\xi$.

For $\eta=0$, choose sets $W_{0}^{(\xi)}, V_{\xi, 0}$ such that

$W_{0}^{(\xi)}, V_{\xi, 0}$ are nonempty, open-and-closed subsets of $S(\mathcal{C})$,

$W_{0}^{(\xi)} \subset \widetilde{w}_{0}^{(\xi)}, V_{\xi, 0} \subset V_{\xi}, W_{0}^{(\xi)} \cap V_{\xi, 0}=\varnothing$.

In choosing such sets, we only need the fact (stated in $1.4(a)$ ), that $S(\mathcal{C}$ ) has no isolated elements.

Let $\eta<\xi$. Assume inductively that, for all $\lambda<\eta$, we have defined sets $W_{\lambda}^{(\xi)}, V_{\xi, \lambda}$ such that

$W_{\lambda}^{(\xi)}, V_{\xi, \lambda}$ are nonempty, open-and-closed subsets of $S(\mathcal{C})$,

$W_{\lambda}^{(\xi)} \subset \widetilde{W}_{0}^{(\xi)}$

$v_{\xi} \supset V_{\xi, 0} \supset \cdots \supset V_{\xi, \lambda} \supset \cdots$,

$W_{\lambda}^{(\xi)} \cap V_{\xi, \lambda}=\varnothing$.

Using $1.4(\mathrm{~b})$, we choose a nonempty, open-and-closed set $\widetilde{V}_{\xi, \eta}$ such that $\widetilde{V}_{\xi, \eta} \subset \bigcap_{\lambda<\eta} V_{\xi, \lambda^{\bullet}}$ We then choose sets $W_{\eta}^{(\xi)}, V_{\xi, \eta}$ such that

$W_{\eta}^{(\xi)}, V_{\xi, \eta}$ are nonempty, open-and-closed subsets of $S(\mathcal{C})$,

$W_{\eta}^{(\xi)} \subset \widetilde{W}_{\eta}^{(\xi)}, V_{\xi, \eta} \subset \widetilde{V}_{\xi, \eta}, W_{\eta}^{(\xi)} \cap V_{\xi, \eta}=\varnothing$

again only using the fact that $S(\mathcal{C})$ has no isolated elements. This completes the subsidiary induction.

Let $W_{\xi}^{(\xi)}$ be any nonempty, open-and-closed subset of $\bigcap_{\eta<\xi} V_{\xi, \eta}$, using once more 1.4(b). It is now clear that the family $\left\{W_{\eta}^{(\xi)}\right.$ : all $\eta$, such that $\left.\eta \leq \xi\right\}$ consists of pairwise disjoint, nonempty, open-and-closed subsets of $S(\mathcal{C})$ for each $\xi<\beta$. This completes the induction.

We have defined nonempty, open-and-closed sets $W_{\eta}^{(\xi)}$ for all $\xi, \eta$, such that $\xi<\beta, \eta \leq \xi$, satisfying the conditions:

$W_{\eta}^{\left(\xi^{\top}\right)} \subset W_{\eta}^{(\xi)}$ for $\xi<\xi^{\prime}<\beta$,

$W_{\eta}^{(\xi)} \subset V_{\eta}$

$\left\{W_{\eta}^{(\xi)}\right.$ : all $\eta$, such that $\left.\eta \leq \xi\right\}$ consists of pairwise disjoint sets for each $\xi<\beta$. 
We employ $1.4(\mathrm{~b})$ once more to find a nonempty, open-and-closed subset $W_{\eta}$ of $\bigcap_{\eta \leq \xi<\beta} W_{\eta}^{(\xi)}$. The family $\left(W_{\xi}\right)_{\xi<\beta}$ satisfies the conditions of the lemma.

For a Boolean algebra $\mathcal{C}$ and an infinite cardinal $\delta$, we set $S(\mathcal{C})_{\lambda}=S(\mathcal{C})_{\times}$ $\{\lambda\}$ for all $\lambda<\delta$. We will identify $\mathcal{C}^{\delta}$ with the Boolean algebra of open-and-closed subsets of $s(\mathcal{C}) \times \delta$. Recall that a set $E \subset \mathcal{C}^{\delta}$ is closed under finite intersection if the intersection of any nonempty finite subset of $E$ is an element of $E$. We prove now the analogue of Lemma $4 \mathrm{C}$ of $\left[\mathrm{K}_{4}\right]$. We note that the proof will use not only the more "elementary" condition 1.4(b), but 1.4(c), as well.

2.2. Lemma. Let $\beta<\alpha$, let $\mathcal{C}$ be an $\alpha$-homogeneous-universal Boolean algebra, let $E$ be a subset of $\mathfrak{B}^{*}(S(\mathcal{C}) \times \delta)$, closed under finite intersection and of cardinality less than $a$. Let

$$
\phi: S_{\omega}(\beta) \rightarrow \mathfrak{B}^{*}(S(\mathcal{C}) \times \delta)
$$

be a monotone function, sucb that $\phi(F) \cap e \neq \varnothing$ for all $F \in S_{\omega}(\beta), e \in E$.

Then, there is a multiplicative function

$$
\psi: S_{\omega}(\beta) \rightarrow \mathcal{B}^{*}(S(\mathcal{C}) \times \delta)
$$

sucb that $\psi(F) \cap e \neq \varnothing$, and $\psi \leq \phi$ for all $F \in S_{\omega}(\beta), e \in E$.

Proof. (a) This part of the proof follows closely the proof of Lemma 4C in $\left[\mathrm{K}_{4}\right]$. We define

$$
\chi: S_{\omega}(\beta) \times E \rightarrow B^{*}(S(\mathcal{C}) \times \delta)
$$

by $\chi(F, e)=\phi(F) \cap e$. We, further, set for $\lambda<\delta$

$$
I_{\lambda}=\left\{\langle F, e\rangle \in S_{\omega}(\beta) \times E: \phi(F) \cap e \cap S(\mathcal{C})_{\lambda} \neq \varnothing\right\} .
$$

Clearly, $\left|S_{\omega}(\dot{\beta}) \times E\right|<\alpha$, and thus $\left|I_{\lambda}\right|<\alpha$. By 2.1 , the family $\chi\left[I_{\lambda}\right]$ admits a disjoint refinement, i.e. there is a family $\left\{\tau_{\lambda}(F, e):\langle F, e\rangle \in I_{\lambda}\right\}$ consisting of nonempty, open-and-closed subsets of $S(\mathcal{C})_{\lambda}$, such that

$$
\begin{aligned}
& { }_{\lambda}(F, e) \cap \tau_{\lambda}\left(F^{\prime}, e^{\prime}\right)=\varnothing \text { for }\langle F, e\rangle \neq\left\langle F^{\prime}, e^{\prime}\right\rangle, \\
& { }_{\tau_{\lambda}}(F, e) \subset \chi^{(}(F, e),
\end{aligned}
$$

where $\langle F, e\rangle,\left\langle F^{\prime}, e^{\prime}\right\rangle \in I_{\lambda}$. Define

$$
\tau: S_{\omega}(\beta) \times E \rightarrow \mathfrak{B}^{*}(S(\mathcal{C}) \times \delta)
$$

by

$$
\tau(F, e)=\bigcup\left\{\tau_{\lambda}(F, e): \text { all } \lambda<\delta \text {, such that }\langle F, e\rangle \in I_{\lambda}\right\}
$$

For $F \in S_{\omega}(\beta)$, set

$$
\Phi(F)=\bigcup\left\{\tau(G, e): G \in S_{\omega}(\beta), G \supset F, e \in E\right\} .
$$


It follows, as in $\left[K_{4}\right]$, that $\Phi$ satisfies the following conditions:

$\Phi(F) \subset \phi(F)$ for $F \in S_{\omega}(\beta)$,

$\Phi\left(F \cup F^{\prime}\right)=\phi(F) \cap\left(F^{\prime}\right)$ for $F, F^{\prime} \in S_{\omega}(\beta)$,

$\Phi(F) \cap e \neq \varnothing$ for $F \in S_{\omega}(\beta), e \in E$.

(b) Remark. In general, we cannot expect $\Phi(F) \cap S(\mathcal{C})_{\lambda}$ to be open-and-closed in $S(\mathcal{C})_{\lambda}$, since $\Phi(F)$ has been defined as a union of less than a pairwise disjoint open-and-closed sets. We may try to correct this difficulty by setting $\psi(F)=$ closure of $\Phi(F)$ in $S(\mathcal{C}) \times \delta$. Then, $\psi$ does satisfy the following conditions:

$$
\begin{aligned}
& \psi(F) \subset \phi(F) \text { for } F \in S_{\omega}(\beta), \\
& \psi\left(F \cup F^{\prime}\right)=\psi(F) \cap \psi\left(F^{\prime}\right) \text { for } F, F^{\prime} \in S_{\omega}(\beta), \\
& \psi(F) \cap e \neq \varnothing \text { for } F \in S_{\omega}(\beta), e \in E .
\end{aligned}
$$

(The second of these conditions holds because of the form of $\Phi$, and by 1.4(c).) However, 1.4(b) clearly shows that $\psi(F)$ is open-and-closed if and only if $\Phi(F)$ is open-and-closed. Hence, we have to do something more than just taking closures.

(c) We complete the proof of the lemma. We begin by noticing the following property of $\Phi$ :

$$
\begin{aligned}
& \text { The set } \Phi(F) \backslash \Phi\left(F^{\prime}\right) \text { is open and of type } \\
& \text { less than } \alpha \text {, for all } F, F^{\prime} \in S_{\omega}(\beta) \text {. }
\end{aligned}
$$

Indeed,

$$
\Phi(F) \backslash \Phi\left(F^{\prime}\right)=\bigcup\left\{r(G, e): G \in S_{\omega}(\beta), G \supset F, e \in E, G \not \supset F^{\prime}\right\} .
$$

We now define mappings

$$
\psi_{i}: \beta \rightarrow \mathfrak{B}^{*}(S(\mathcal{C}) \times \delta), \quad 1 \leq i<\omega .
$$

Let $\psi_{1}(\xi)=\phi(\{\xi\})$. Inductively, suppose that we have defined

$$
\psi_{j}: \beta \rightarrow \mathfrak{B}^{*}(S(\mathcal{C}) \times \delta), \quad 1 \leq j \leq i,
$$

where $1 \leq i$, such that

(i) $\bar{\Phi}(\{\xi\}) \subset \psi_{i}(\xi) \subset \psi_{i-1}(\xi) \subset \cdots \subset \psi_{1}(\xi)=\phi(\{\xi\})$, and

(ii) if $|F| \leq i$, say $F=\left\{\xi_{1}, \ldots, \xi_{i}\right\}$, then $\Phi(F) \subset \psi_{i}\left(\xi_{1}\right) \cap \ldots \cap \psi_{i}\left(\xi_{i}\right) \subset \phi(F)$.

We define $\psi_{i+1}: \beta \rightarrow \mathscr{R}^{*}(S(\mathcal{C}) \times \delta)$. For $\xi<\beta$, set

$$
S_{i+2}(\beta, \xi)=\left\{F \in S_{\omega}(\beta):|F| \leq i+1, \xi \in F\right\} .
$$

Let $F \in S_{i+2}(\beta, \xi)$. If $|F|<i+1$, set $\psi_{i+1}^{F}(\xi)=\psi_{i}(\xi)$. If $|F|=i+1$, say $F=$ $\left\{\xi, \xi_{1}, \ldots, \xi_{i}\right\}$, we proceed as follows. Let

$$
\begin{aligned}
R(F)= & \left\{\psi_{i}(\xi) \cap \psi_{i}\left(\xi_{1}\right) \cap \cdots \cap \psi_{i}\left(\xi_{i}\right)\right\} \backslash \\
& \left\{\psi_{i}(\xi) \cap \psi_{i}\left(\xi_{1}\right) \cap \cdots \cap \psi_{i}\left(\xi_{i}\right) \cap \phi\left(\left\{\xi, \xi_{1}, \cdots, \xi_{i}\right\}\right)\right\} .
\end{aligned}
$$


Thus, $R(F)$ is open-and-closed in $S(\mathcal{C}) \times \delta$. Let $F^{(j)}=\{A: A \subset F,|A|=j\}$, where $1 \leq j \leq i$. Note, at first, that $R(F) \cap \Phi(F)=\varnothing$, since $\Phi(F) \subset \phi(F)$. Set $\Sigma(F)=$ $\varnothing$, and $\Sigma(A)=R(F) \cap \Phi(A)$, for $A \in F^{(i)}$. Clearly, $\left\{\Sigma(A): A \in F^{(i)}\right\}$ is a finite family of pairwise disjoint sets. For $A \in F^{(i-1)}$, set $\Sigma(A)=R(F) \cap$ $\left(\Phi(A) \backslash \bigcup\left\{\Phi(B): B \in F^{(i)}\right\}\right)$, etc.; by finite induction. Thus, we have defined sets $\Sigma(A)$ for all $A \in S_{\omega}(F)$, such that $\Sigma(A) \cap \Sigma(B)=\varnothing$ if $A \neq B$,

$\Sigma(A) \subset R(F)$,

$\Sigma(A)$ is an open subspace of $S(\mathcal{C}) \times \delta$, such that $\Sigma(A) \cap S(\mathcal{C})_{\lambda}$ is of type less than $\alpha$, for all $\lambda<\delta$,

$$
R(F) \cap \Phi(\{\xi\})=\bigcup\{\Sigma(A): \xi \in A\} .
$$

(The third property follows from (1) and the fact that $R(F)$ is open-and-closed.) We use $1.4(c)$ to find open-and-closed sets $V(A)$, for $A \in S_{\omega}(F)$, such that

$V(A) \cap V(B)=\varnothing$ if $A \neq B$,

$\Sigma(A) \subset V(A) \subset R(F)$,

$V(A)=\varnothing$ if $\Sigma(A)=\varnothing$, and thus in particular $V(F)=\varnothing$. We set $R_{\xi}^{F}=$ $\bigcup\left\{V(A): A \in S_{\omega}(F), \xi \in F\right\}$, and

$$
\begin{aligned}
\psi_{i+1}^{F}(\xi)= & \left\{\psi_{i}(\xi) \backslash\left(\psi_{i}(\xi) \cap \psi_{i}\left(\xi_{1}\right) \cap \ldots \cap \psi_{i}\left(\xi_{i}\right)\right)\right\} \\
& \cup\left\{\psi_{i}(\xi) \cap \psi_{i}\left(\xi_{1}\right) \cap \ldots \cap \psi_{i}\left(\xi_{i}\right) \cap \phi(F)\right\} \cup R_{\xi^{\bullet}}^{F}
\end{aligned}
$$

Note that $\psi_{i+1}^{F}(\xi)$ is open-and-closed in $S(\mathcal{C}) \times \delta$, and that $\Phi(\{\xi\}) \subset \psi_{i+1}^{F}(\xi)$ for all $F \in S_{i+2}(\beta, \xi)$. We use once more $1.4(\mathrm{c})$ to find an open-and-closed set $\psi_{i+1}(\xi)$ of $S(\mathcal{C}) \times \delta$, such that

$$
\Phi(\{\xi\}) \subset \psi_{i+1}(\xi) \subset \bigcap\left\{\psi_{i+1}^{F}(\xi): F \in S_{i+2}(\beta, \xi)\right\},
$$

since $\left|S_{i+2}(\beta, \xi)\right|=\beta<\alpha$. This completes the definition of $\psi_{i+1}$. We now verify that $\psi_{i+1}$ satisfies the inductive conditions (i) and (ii). Condition (i) is obvious. For (ii), let $|F| \leq i+1$. If $|F|<i+1$, say $F=\left\{\xi_{1}, \ldots, \xi_{i}\right\}$ (with possible repetitions), then

$$
\Phi(F) \subset \psi_{i+1}\left(\xi_{1}\right) \cap \cdots \cap \psi_{i+1}\left(\xi_{i}\right) \subset \psi_{i}\left(\xi_{1}\right) \cap \cdots \cap \psi_{i}\left(\xi_{i}\right) \subset \phi(F),
$$

using the facts that $\Phi$ is multiplicative, and that $\psi_{i+1} \leq \psi_{i}$. Let now $|F|=i+1$, say $F=\left\{\xi_{1}, \ldots, \xi_{i+1}\right\}$. Then

$$
\begin{aligned}
\psi_{i+1}\left(\xi_{1}\right) \cap & \cap \cap \psi_{i+1}\left(\xi_{i+1}\right) \subset \psi_{i+1}^{F}\left(\xi_{1}\right) \cap \cdots \cap \psi_{i+1}^{F}\left(\xi_{i+1}\right) \\
& \subset\left(\psi_{i}\left(\xi_{1}\right) \cap \cdots \cap \psi_{i}\left(\xi_{i+1}\right) \cap \phi(F)\right) \cup\left\{R_{\xi_{1}}^{F} \cap \cdots \cap R_{\xi_{i+1}}^{F}\right\}
\end{aligned}
$$


Let $p \in R_{\xi}^{F} \cap \cdots \cap R_{\xi_{i+1}}^{F}$; then, $p \in V(A)$ for some unique $A \in S_{\omega}(F)$. Hence, $\xi_{1}, \ldots, \xi_{i+1} \in A$, i.e., $A=F$. But $\Sigma(F)=V(F)=\varnothing$. Thus, $R_{\xi_{1}}^{F} \cap \ldots \cap R_{\xi_{i+1}}^{F}$ $=\varnothing$. It now follows that $\psi_{i+1}\left(\xi_{1}\right) \cap \cdots \cap \psi_{i+1}\left(\xi_{i+1}\right) \subset \phi(F)$, proving (ii).

To complete the proof of the lemma, use once more 1.4 (c) to find an open-andclosed set $\psi(\{\xi\})$ in $S(\mathcal{C}) \times \delta$, such that

$$
\Phi(\{\xi\}) \subset \psi(\{\xi\}) \subset \bigcap\left\{\psi_{i}(\xi): 1 \leq i<\omega\right\} .
$$

Define $\psi: S_{\omega}(\beta) \rightarrow B^{*}(S(\mathcal{C}) \times \delta)$ by $\psi(F)=\bigcap_{\xi \in F} \psi(\{\xi\})$. It is clear that $\psi$ satisfies the conditions of the lemma.

The main result of this section can now be obtained.

Theorem A. Let $\alpha=a^{a}>\omega, \omega \leq \delta<\alpha$, and let $\mathcal{S}_{\alpha}$ denote the a-bomogene. ous-universal Boolean algebra of cardinality $\alpha$. There is a countably incomplete, $\delta$-uniform, and a-good ultrafilter of open-and-closed subsets of $S\left(\delta_{\alpha}\right) \times \delta$.

Proof. Note that $\left|\mathfrak{B}^{*}\left(S\left(\mathcal{S}_{\alpha}\right) \times \delta\right)\right|=\left|\mathcal{S}_{\alpha}^{\delta}\right|=a^{\delta}=\alpha$, and hence that there are, at most, $\alpha^{\beta}$ monotone maps from $S_{\omega}(\beta)$ to $\mathfrak{B}^{*}\left(S\left(\delta_{\alpha}\right) \times \delta\right)$, for $\beta$ infinite; thus, there are, at most, $\alpha^{a}=\alpha$ monotone maps from $S_{\omega}(\beta)$ to $\mathfrak{B}^{*}\left(S\left(\mathcal{S}_{\alpha}\right) \times \delta\right)$, as $\beta$ varies over all infinite cardinals less than $\alpha$. In view of Lemmas 2.1 and 2.2, the proof of the theorem follows the argument given by Keisler in his proof of Theorem 4.4 in $\left[K_{4}\right]$.

2.3. Remark. For an ultrafilter $p$ of open-and-closed subsets of a space, let $G(p)$ be the least cardinal $\gamma$, such that $p$ is not $\gamma$-good (if such a cardinal exists). (This is Keisler's notion of the degree of goodness of $p$.) It is proved, in Theorem $A$, that there is an ultrafilter $p$ of open-and-closed subsets of $S\left(\delta_{a}\right) \times \delta$, such that $G(p) \geq a$. I have not been able to settle whether $G(p)=\alpha$. It is likely that this is so, and that the ideas involved in the proof of Theorem 3.7 in $\left[\mathbf{N}_{1}\right]$ are relevant in this connection. This problem is related to Keisler's problem no. 2 in $\left[K_{4}\right]$.

3. Adequate ultrafilters on products of special Boolean algebras. In this section we extend the methods of $\$ 2$ to the larger class of special Boolean algebras. The main result (Theorem $B$ ) establishes the existence of adequate ultrafilters on certain products of special Boolean algebras.

3.1. Lemma. Let $\beta, \delta$ be infinite cardinals, sucb that $\delta \leq \beta$. Let $\mathcal{C}_{0}$ be a Boolean algebra, such that $\left|\mathcal{C}_{0}\right| \leq 2^{\beta}$, and let $E_{0}$ be a family of nonempty, open. and-closed subsets of $S\left(\mathcal{C}_{0}\right) \times \delta$ such that $E_{0}$ is a filter, and $S\left(\mathcal{C}_{0}\right) \times \delta \notin E_{0}$ for all $D \in S_{\delta}(\delta)$. Then, there is a Boolean algebra embedding $b: \mathcal{C}_{0} \rightarrow \mathcal{C}_{1}$, and there is an ultrafilter $p_{1}$ of open-and-closed subsets of $s\left(\mathcal{C}_{1}\right) \times \delta$, such that

$p_{1}$ is $\delta$-uniform and countably incomplete,

$\left(S(b) \times \mathrm{id}_{\delta}\right)^{-1}\left[E_{0}\right] \subset p_{1}$, and

$p_{1}$ is $\left(\beta^{+}, E_{0}\right)$-good. 
Proof. Using 1.2, we embed $\mathcal{C}_{0}$ into a $\left(2^{\beta}\right)^{+}$-homogeneous-universal Boolean algebra $\mathcal{C}$ of cardinality $2^{2^{\beta}}, b_{0}: \mathcal{C}_{0} \rightarrow \mathcal{C}$. Note that $|E| \leq\left(2^{\beta}\right)^{\delta}=2^{\beta}$, and, thus,

$$
\left|\operatorname{Mon}\left(\beta^{+}, E_{0}\right)\right| \leq\left(2^{\beta}\right)^{\beta}=2^{\beta} \text {. }
$$

We transfer all considerations to $\mathcal{C}$ : Let

$$
\begin{aligned}
& E_{0}^{*}=\left(S\left(b_{0}\right) \times \mathrm{id}_{\delta}\right)^{-1}\left[E_{0}\right], \\
& \phi^{*}=\operatorname{Mon}\left(\beta^{+}, b_{0}\right) \phi \text { for } \phi \in \operatorname{Mon}\left(\beta^{+}, E_{0}\right) .
\end{aligned}
$$

Well-order $\operatorname{Mon}\left(\beta^{+}, E_{0}\right):\left\{\phi_{\eta}\right\}_{\eta<2} \beta$, and let $\left\{\phi_{\eta}^{*}\right\}_{\eta<2} \beta$ be the corresponding wellordering at $\mathcal{C}$. We proceed by transfinite induction on $2^{\beta}$, using 2.2 at every stage (with $\alpha=\left(2^{\beta}\right)^{+}$). We first adjoin to $E_{0}^{*}$ the family $\{S(\mathcal{C}) \times(\delta-D): D \epsilon$ $\left.S_{\delta}(\delta)\right\}$, and call the resulting family $D_{0}$.

For $0<\zeta<2^{\beta}$, $\zeta$ a limit ordinal, we set $D_{\zeta}=\bigcup_{\eta<\zeta} D_{\eta}$.

We define $D_{\eta+1}$ for all $\eta<2^{\beta}$. Let $\phi_{\eta}^{*}: S_{\omega}(\gamma) \rightarrow E_{0}^{*}$, for some cardinal $\gamma$ (depending on $\eta$ ), $\gamma \leq \beta$. We apply Lemma 2.2, to find a multiplicative mapping $\psi_{\eta}^{*}: S_{\omega}(\gamma) \rightarrow \mathfrak{B}^{*}(S(\mathcal{C}) \times \delta)$, such that

$\psi_{\eta}^{*} \leq \phi_{\eta}^{*}$, and

$\psi_{\eta}^{*}(F) \cap d \neq \varnothing$ for all $F \in S_{\omega}(\gamma), d \in D_{\eta}$.

Let $D_{\eta+1}$ be the smallest subfamily of $\mathfrak{B}^{*}(S(\mathcal{C}) \times \delta)$, containing

$$
D_{\eta} \cup\left\{\psi_{\eta}^{*}(F) \cap d: F \in S_{\omega}(\gamma), d \in D_{\eta}\right\}
$$

and closed under finite intersection. Inductively, it is easy to prove that $\left|D_{\eta}\right| \leq 2^{\beta}$ for all $\eta<2^{\beta}$, and that $D_{\eta}$ consists of nonempty (open-and-closed) subsets of $\mathcal{B}^{*}(S(\mathcal{C}) \times \delta)$. Let $E_{1}^{*}=\bigcup_{\eta<2} D_{\eta}^{\eta}$; thus $\left|E_{1}^{*}\right| \leq 2^{\beta}$. Let $\mathcal{C}_{1}$ be a Boolean subalgebra of $\mathcal{C}$, having cardinality $2^{\beta}$, and containing $\mathcal{C}_{0}$ and all elements of $\mathcal{C}$ of the from $e \cap(S(\mathcal{C}) \times\{\xi\})$ for all $e \in E_{1}^{*}, \xi<\delta$. Let $p_{1}$ be an ultrafilter of open-and-closed subsets of $S\left(\mathcal{C}_{1}\right) \times \delta$, containing $E_{1}^{*}$. The conditions of the lemma are, clearly, satisfied.

By a repeated use of 3.1 and 1.2 we obtain the following result.

3.2. Lemma. Let $\beta, \delta$ be infinite cardinals, sucb that $\delta<\beta$. Let $\mathcal{C}$ be a Boolean algebra, such that $\left|\mathcal{C}_{0}\right| \leq 2^{\beta}$, and let $E_{0}$ be a family of nonempty, openand-closed subsets of $S\left(\mathcal{C}_{0}\right) \times \delta$, such that $E_{0}$ is a filter, and $s\left(\mathcal{C}_{0}\right) \times D \notin E_{0}$ for all $D \in S_{\delta}(\delta)$. Then, there is a Boolean algebra $\mathcal{C}$, such that

$$
\begin{aligned}
& |\mathcal{C}|=2^{\beta}, \\
& \mathcal{C} \text { is } \beta^{+} \text {-homogeneous-universal, }
\end{aligned}
$$

there is a Boolean algebra embedding $b: \mathcal{C}_{0} \rightarrow \mathcal{C}$, and there is an ultrafilter $p$ of open-and-closed subsets of $S(\mathcal{C}) \times \delta$, such that

$p$ is countably incomplete and $\delta$-uniform, 
$\left(S(b) \times \mathrm{id}_{\delta}\right)^{-1}\left[E_{0}\right] \subset p$, and $p$ is $\beta^{+}$-good.

Proof. Using 1.2, we embed $\mathcal{C}_{0}$ into a $\left(2^{\beta}\right)^{+}$-homogeneous-universal Boolean algebra $\mathfrak{D}$ of cardinality ${ }_{2}^{2}{ }^{\beta}$. We may assume that $\mathcal{C}_{0} \subset \mathcal{D}$.

The proof proceeds by transfinite induction on $\beta^{+}$. With no loss of generality, we may assume that $E_{0}$ contains the family $\left\{S(\mathcal{C}) \times(\delta / D): D \in S_{\delta}(\delta)\right\}$. We start with $\mathcal{C}_{0}, E_{0}$.

Let $\xi<\beta^{+}$. Assume inductively that for all $\zeta<\xi$ we have defined Boolean subalgebras $\mathcal{C}_{\zeta}$ of $\mathscr{D}$, such that $\left|\mathcal{C}_{\zeta}\right|=2^{\beta}$, and ultrafilters $p_{\zeta}$ of open-and-closed subsets of $S\left(\mathcal{C}_{\zeta}\right) \times \delta$, and that for all $\zeta<\eta<\xi$ we have defined Boolean algebra embeddings $b_{\zeta, \eta}: C_{\zeta} \rightarrow C_{\eta}$, in such a way that for all $\zeta<\xi$, a direct system over $\zeta$ is formed, and in addition $\left(S\left(b_{\zeta, \eta}\right) \times \mathrm{id}_{\delta}\right)^{-1}\left[p_{\zeta}\right] \subset p_{\eta}$.

If $\xi$ is a limit ordinal, let $\mathcal{C}_{\xi}$ be the direct limit of the already existing direct system (over $\xi$ ), and let $b_{\zeta, \xi}: \mathcal{C}_{\zeta} \rightarrow \mathcal{C}_{\xi}$ be the induced injections for $\zeta<\xi$. Also, let $p_{\xi}$ be the direct limit of the corresponding direct system of the ultrafilters $\left\{p_{\zeta}: \zeta<\xi\right\}$ with connecting maps given by $\left(S\left(b_{\zeta, \eta}\right) \times \mathrm{id}_{\delta}\right)^{-1}: p_{\zeta} \rightarrow p_{\eta}$ for $\zeta<\eta<\xi$. It is clear that $p_{\xi}$ is an ultrafilter of open-and-closed subsets of $s\left(\mathcal{C}_{\xi}\right) \times \delta$.

Let $\xi=\zeta+1$. We use Lemma 3.2, with $\mathcal{C}_{\zeta}, p_{\zeta}$, in place of $\mathcal{C}_{0}, E_{0}$. This gives a Boolean algebra $\mathcal{C}_{\xi, 0}$, such that $\left|\mathcal{C}_{\xi, 0}\right|=2^{\beta}$, a Boolean algebra embedding $b_{\zeta, \xi, 0}: \mathcal{C}_{\zeta} \rightarrow \mathcal{C}_{\xi, 0}$, and an ultrafilter $p_{\xi, 0}$, satisfying the conditions of 3.2 , in the context of our present notation. Using the $\left(2^{\beta}\right)^{+}$-homogeneity, $\left(2^{\beta}\right)^{+}$-universality of $\mathscr{D}$, in the usual way, we may assume that $\mathcal{C}_{\zeta} \subset \mathcal{C}_{\xi, 0} \subset \mathcal{D}$ and that $b_{\zeta, \xi, 0}$ is the indicated inclusion. We, now, use 1.2 to embed $\mathcal{C}_{\xi, 0}$ in a $\beta^{+}$-homogeneous-universal Boolean algebra $\mathcal{C}_{\xi}$ of cardinality $2^{\beta}$. Using the $\left(2^{\beta}\right)^{+}$-homogeneity, $\left(2^{\beta}\right)^{+}$-universality of $\mathscr{D}$, in the usual way, we may assume that $\mathcal{C}_{\xi, 0} \subset$ $\mathcal{C}_{\xi} \subset \mathcal{D}$. Let $b_{\xi, 0}$ be the inclusion $\mathcal{C}_{\xi, 0} \subset \mathcal{C}_{\xi}$. Let $b_{\zeta, \xi}=b_{\xi, 0}{ }^{\circ} b_{\zeta, \xi, 0}$, and let $p_{\xi}$ be any ultrafilter of open-and-closed subsets of $S\left(\mathcal{C}_{\xi}\right) \times \delta$, containing $\left(s\left(b_{\xi, 0}\right) \times \mathrm{id}_{\delta}\right)^{-1}\left[p_{\xi, 0}\right]$. This completes the inductive definition.

We let $\mathcal{C}$ be the direct limit of the direct system $\left\{\mathcal{C}_{\xi}: \xi<\beta^{+}\right\}$, with connecting maps $b_{\zeta, \xi}, \zeta<\xi<\beta^{+}$, and let $p$ be the direct limit of the corresponding direct system $\left\{p_{\xi}: \xi<\beta^{+}\right\}$, with connecting maps $\left(S\left(b_{\zeta, \xi}\right) \times \mathrm{id}_{\delta}\right)^{-1}, \zeta<\xi<\beta^{+}$. Clearly, $p$ is an ultrafilter of open-and-closed subsets of $S(\mathcal{C}) \times \delta$. Further, it follows inductively that $\left|\mathcal{C}_{\xi}\right|=2^{\beta}, \xi<\beta^{+}$, and hence $|\mathcal{C}|=2^{\beta}$. By the regularity of the cardinal $\beta^{+}$, it is clear, using the familiar chain arguments, that $\mathcal{C}$ is $\beta^{+}$homogeneous-universal and that $p$ is $\beta^{+}$-good, countably incomplete and $\delta$-uniform.

We are now in a position to prove the main result of this section.

Theorem B. Let $a=2^{a}>\omega, \omega \leq \delta<\alpha$, and let $\mathcal{S}_{\alpha}$ denote the unique special 
Boolean algebra of cardinality $\alpha$. Then, $\mathcal{S}_{a}$ has a specializing chain $\left\{\mathcal{C}_{\beta}: \beta<\alpha\right\}$, sucb that $\left|\mathcal{C}_{\beta}\right|=2^{\beta}$ for $\beta<\alpha$, (and $\mathcal{C}_{\beta}$ is $\beta^{+}$-homogeneous-universal for $\beta<\alpha$ ), and an ultrafilter $p$ of open-and-closed subsets of $S\left(\mathcal{S}_{\alpha}\right) \times \delta$ which is adequate, i.e., one that satisfies the following conditions:

$p$ is countably incomplete and $\delta$-uniform, and

$p_{\beta}=p \cap \mathfrak{B}^{*}\left(S\left(\mathcal{C}_{\beta}\right) \times \delta\right)$ is $\beta^{+}$-good for all $\beta, \delta \leq \beta<a$.

Proof. Let $\left\{\mathscr{D}_{\beta}: \beta<\alpha\right\}$ be a specializing chain for $\int_{\alpha}$, such that $\left|D_{\beta}\right|=2^{\beta}$ for $\beta<\alpha$. (This is possible because of exercise $6 \mathrm{~J}$ in [CK] and the fact that a $\beta^{+}$-homogeneous-universal Boolean algebra must be of cardinality at least $2^{\beta}$, because of the corresponding statement for $\eta_{\beta^{+}}{ }^{- \text {sets }}\left[\mathrm{H}_{2}\right]$ and Corollary 1.2 in $\left[\mathrm{N}_{1}\right]$.) Let $\mathcal{C}_{\beta}=\mathfrak{D}_{\beta}$ for $\beta<\delta$.

Let $\delta \leq \beta<\alpha$. Assume inductively that for every cardinal $\gamma, \omega \leq \gamma<\beta$, we have defined Boolean algebras $\mathcal{C}_{\gamma}$, such that

$\left|\mathcal{C}_{\gamma}\right|=2^{\gamma}$ for $\gamma<\beta$,

$\mathcal{C}_{\gamma}$ is $\gamma^{+}$-homogeneous-universal for $\gamma<\beta$,

$\mathcal{C}_{\gamma_{1}} \subset \mathcal{C}_{\gamma_{2}}$ for $\gamma_{1}<\gamma_{2}<\beta$,

and that we have defined ultrafilters $p_{y}$ of open-and-closed subsets of $s\left(\mathcal{C}_{\gamma}\right) \times \delta$, such that

$$
p_{\gamma_{1}}=p_{\gamma_{2}} \cap B^{*}\left(s\left(\mathcal{C}_{\gamma_{1}}\right) \times \delta\right) \text { for } \gamma_{1}<\gamma_{2}<\beta \text {, }
$$

$p_{\gamma}$ is countably incomplete and $\delta$-uniform for $\gamma<\beta$, and

$p_{y}$ is $\gamma^{+}$-good for $\delta \leq \gamma<\beta$.

We now define $\mathcal{C}_{\beta}, p_{\beta}$ as follows. Let $\widetilde{C}_{\beta}=\bigcup_{\gamma<\beta} C_{\gamma}$ and $\tilde{p}_{\beta}=\bigcup_{\gamma<\beta} p_{\gamma}$. Clearly, $\left|\widetilde{C}_{\beta}\right| \leq 2^{\beta}$, and $\tilde{p}_{\beta}$ is a filter of open-and-closed subsets of $s\left(\widetilde{C}_{\beta}\right) \times \delta$, which is countably incomplete and $\delta$-uniform. Thus, Lemma 3.2 applies. Let $\mathcal{C}_{\beta}, p_{\beta}$ be the Boolean algebra and the $\left(\beta^{+}\right.$-good) ultrafilter, respectively, that satisfy the conclusion of 3.2 , in the context of our present notation. This completes the inductive definition of the (specializing) chain $\left\{C_{\beta}, \beta<\alpha\right\}$. Thus $\bigcup_{\beta<\alpha} \mathcal{C}_{\beta}$ is isomorphic to $\delta_{\alpha}$. Let $p=\bigcup_{\beta<\alpha} p_{\beta}$. It is clear that $p$ is a countably. incomplete and $\delta$-uniform ultrafilter of open-and-closed sets of $S\left(\delta_{\alpha}\right) \times \delta$, which is adequate.

It is easy to see that if, in addition, $\alpha$ is regular, i.e., if $a=\alpha^{(\infty)}>\omega$, then the adequate ultrafilter $p$ of Theorem B is, in fact, $\alpha$-good. Thus, Theorem A may be considered as a special case of Theorem $B$.

\section{PART II: COMPLETIONS}

4. Good ultrafilters on $\alpha$-completions of $\alpha$-homogeneous-universal Boolean algebras. We will prove in this section the existence of countably incomplete, $\alpha$-good ultrafilters on the $a$-completion of the $\alpha$-homogeneous-universal Boolean algebra of cardinality a (Theorem C). Our first step is to identify this $a$-com- 
pletion with an $\alpha$-complete field of sets $\alpha$-generated by the $\alpha$-homogeneous-universal Boolean algebra. For this purpose the following notion of the $\pi_{a}$-topology is helpful (cf. $\$ 3$ in $\left[\mathbf{N}_{\mathbf{l}}\right]$ ). For any Boolean algebra $\mathcal{C}$, and any infinite cardinal $a$, we denote by $\pi_{a}\left(S(\mathcal{C})\right.$ ) the topology (referred to as the $\pi_{a}$-topology) on the Stone space $S(\mathcal{C})$ of $\mathcal{C}$ determined by the (canonical) base for open sets consisting of the family of all subsets of $S(\mathcal{C})$ of the form $\bigcap_{\xi<\beta} V_{\xi}$, where $\beta<\alpha$ and $\left(V_{\xi}\right)_{\xi<\beta}$ is a family of elements of $\mathcal{C}$. It is clear, since this family is closed under finite intersections, that indeed it constitutes a base for a topology which is finer (= larger) than the original topology of $S(\mathcal{C})$.

4.1. Lemma. If $a$ is an infinite regular cardinal, then the Boolean algebra $\left.\mathfrak{B}_{a}(S(\mathcal{C}))\right)$ is an a-complete field of subsets of $S(\mathcal{C})$.

Proof. Let $\beta<\alpha$ and let $\left(X_{\xi}\right)_{\xi<\beta}$ be a family of open-and-closed sets in $\pi_{\alpha}(S(\mathcal{C}))$. Then, of course, $X=\bigcup_{\xi<\beta}^{5} X_{\xi}$ is open. To prove that $X$ is closed, let $p \notin X$. For every $\xi<\beta$, let $T_{\xi}$ be a neighborhood of $p$ in the $\pi_{a}$-topology, such that $T_{\xi} \cap X_{\xi}=\varnothing$. We may assume that $T_{\xi}$ is a basic $\pi_{a}$ oneighborhood, i.e., the intersection of, say, $\gamma_{\xi}, \gamma_{\xi}<\alpha$, open-and-closed subsets of $S(\mathcal{C})$. Then $p \in T=\bigcap_{\xi<\beta} T_{\xi}, T \cap X=\varnothing$, and $T$ is the intersection of at most $\gamma=$ $\Sigma_{\xi<\beta} \gamma_{\xi}$ open-and-closed subsets of $S(\mathcal{C})$. Since $\alpha$ is regular, $\gamma<\alpha$, i.e., $T$ is $\pi_{a}$ oppen; hence $X \in \mathfrak{B}\left(\pi_{a}(S(\mathcal{C}))\right)$.

4.2. Lemma. Let $a$ be an infinite regular cardinal, and let $\mathcal{C}$ be an $a-b o$ mogeneous-universal Boolean algebra. Then the a-completion $\mathcal{C}^{(a)}$ of $\mathcal{C}$ is isomorpbic to the a-complete field of subsets of $S(\mathcal{C})$ a-generated by $\mathcal{C}$.

Proof. Let $\mathcal{F}^{(a)}$ be the $a$-complete field of subsets of subsets of $S(\mathcal{C})$ a-generated by $\mathcal{C}$. By 1.6 , we only must show that $\mathcal{F}^{(\alpha)}$ contains $\mathcal{C}$ as a dense subalgebra. It is clear, by 4.1 , that $C \subset \mathcal{F}^{(a)} \subset \mathcal{B}\left(\pi_{a}(S(\mathcal{C}))\right)$. We prove that $\mathcal{C}$ is dense in $\mathfrak{B}\left(\pi_{a}(S(\mathcal{C}))\right)$. Indeed, let $V \in \mathfrak{B}\left(\pi_{a}(S(\mathcal{C}))\right), V \neq \varnothing$. Since $V$ is, in particular, an open subset in $\pi_{\alpha}$-topology, $V$ contains a nonempty set $T$ of the canonical base for the $\pi_{a}$-topology, say $T=\bigcap_{\xi<\beta} V_{\xi}$, where $\beta<\alpha$ and $V_{\xi} \in \mathcal{C}, V_{\xi} \neq \varnothing$ for $\xi<\beta$. Since $T$ is nonempty, by $1.4(\mathrm{~b})$, there is $W \in C, W \neq \varnothing$, and $W \subset T$. In particular, $\varnothing \neq W \subset V$, showing that $\mathcal{C}$ is dense in $\mathcal{B}\left(\pi_{a}(S(\mathcal{C}))\right.$ ), and hence in $\mathfrak{F}^{(a)}$.

We are now in a position to use 2.1 , in conjunction with 4.2 , to prove the "disjoint refinement" lemma for $a$-completions of $a$-homogeneous-universal Boolean algebras.

4.3. Lemma. Let $\alpha$ be an infinite regular cardinal, let $\beta<\alpha$, let $\mathcal{C}$ be an a-bomogeneous-universal Boolean algebra, and let $\left(V_{\xi}\right)_{\xi<\beta}$ be a family of non- 
empty elements of $\mathcal{C}^{(\alpha)}$. Then, there is a family $\left(W_{\xi}\right)_{\xi<\beta}$ of nonempty elements of $\mathcal{C}$, such that $W_{\xi} \subset V_{\xi}$ for $\xi<\beta$, and $W_{\xi} \cap W_{\zeta}=\varnothing$ for $\xi<\zeta<\beta$.

Proof. By 4.2, we identify $\mathcal{C}^{(a)}$ with the $a$-complete field of subsets of $s(\mathcal{C}) \alpha \cdot$ generated by $\mathcal{C}$. Since $\mathcal{C}$ is dense in $\mathcal{C}^{(\alpha)}$, for every $\xi<\beta$, there is $D_{\xi} \in C$, such that $\varnothing \neq D_{\xi} \subset V_{\xi}$. We apply 2.1 to the family $\left(D_{\xi}\right)_{\xi<\beta}$, to obtain a family $\left(W_{\xi}\right)_{\xi<\beta}$ in $\mathcal{C}$, such that $\varnothing \neq W_{\xi} \subset D_{\xi}$ for $\xi<\beta$ and $W_{\xi} \cap W_{\zeta}=\varnothing$ for $\xi<\zeta<\beta$. This family satisfies the conditions of the lemma.

We now prove without difficulty results corresponding to 2.2 and Theorem A. Recall that $\mathcal{C}^{(\alpha) *}$ denotes $\mathcal{C}^{(\alpha)} \backslash\{\varnothing\}$.

4.4. Lemma. Let $\alpha$ be an infinite regular cardinal, let $\beta<\alpha$, and let $\mathcal{C}$ be an a-bomogeneous-universal algebra. Let $E \subset C^{(\alpha) *}$ be closed under finite intersections. Furtber, let $\phi: S_{\omega}(B) \rightarrow \mathcal{C}^{(\alpha) *}$ be a monotone mapping, such that $\phi(F) \cap e \neq \varnothing$ for all $F \in S_{\omega}(B), e \in E$. Then, there is a multiplicative mapping $\psi: S_{\omega}(B) \rightarrow \mathcal{C}^{(a) *}$ sucb that $\psi \leq \phi$, and $\psi(F) \cap e \neq \varnothing$ for all $F \in S_{\omega}(\beta)$, e $\in E$.

Proof. It will be sufficient to make the following remarks. We have available the "disjoint refinement" Lemma 4.3; the standard proof of Keisler (Lemma $4 \mathrm{C}$ in $\left[\mathbf{K}_{4}\right]$ ) employs a corresponding "disjoint refinement" lemma, and the definition of $\psi$ is given, using only unions of families of cardinalities less than a (cf. parts (a) and (b) of the proof of Lemma 2.2 above); since, by 4.2, we have identified $\mathcal{C}^{(a)}$ with the a-complete field of subsets of $S(\mathcal{C})$ a-generated by $\mathcal{C}$, the standard proof can be carried intact to the present situation.

Theorem C. Let $\alpha=\alpha^{a}>\omega$ and let $S_{a}$ be the $\alpha$-homogeneous-universal Boolean algebra of cardinality $a$. There is a countably incomplete, a-good ultrafilter on $\mathfrak{S}_{a}^{(\alpha)}$.

Proof. By 1.5, $\left|\mathcal{S}_{a}^{(a)}\right|=\alpha$. Hence, there are at most $\alpha^{a}=\alpha$ monotone maps from $S_{\omega}(\beta)$ into $S_{a}^{(\alpha)}$, as $\beta$ varies over all cardinals less than $\alpha$. The proof of Theorem 4.4 in $\left[K_{4}\right]$ can be repeated here, with no changes.

For future use, we outline the proof of the following analogue of Lemma 4B in $\left[\mathrm{K}_{4}\right]$.

4.5. Lemma. Let $\alpha$ be an infinite regular cardinal, and let $\mathcal{C}$ be an $a$-bomogeneous-universal Boolean algebra. Let $E \subset \mathcal{C}^{(a) *}$ be such that

$|E|<\alpha$, and

$E$ is closed under finite intersection.

Then, there is $D \subset \bigodot^{(\alpha) *}$, such that

$|D|<a$,

$D$ is closed under finite intersection, and

$D$ contains a sequence $\left(Y_{n}\right)_{n<\omega}$, such that $\bigcap_{n<\omega} Y_{n}=\varnothing$. 
Proof. Let $|E|=\beta<\alpha$ and let $\left(E_{\xi}\right)_{\xi<\beta}$ be a well-ordering of $E$. By 4.3, there is a family $\left(K_{\xi}\right)_{\xi<\beta} \subset \mathcal{C}^{*}$, such that

$K_{\xi} \subset E_{\xi}$ for $\xi<\beta$, and

$K_{\xi} \cap K_{\zeta}=\varnothing$ for $\xi<\zeta<\beta$.

Thus $K_{\xi}$ is a nonempty open-and-closed subset of $S(\mathcal{C})$, and there is no difficulty in finding elements $X_{\xi, n}, \xi<\beta, n<\omega$, of $\mathcal{C}^{(\alpha) *}$, such that

$$
\begin{aligned}
& K_{\xi}=X_{\xi, 0} \supset \ldots \supset X_{\xi, n} \supset \ldots \text {, and } \\
& \bigcap_{n<\omega} X_{\xi, n}=\varnothing \text { for } \xi<\beta .
\end{aligned}
$$

Let $Y_{n}=\bigcup_{\xi<\beta}^{\xi, n} X_{\xi, n}$ for $n<\omega$. Then, $Y_{n} \in \mathcal{C}^{(\alpha) *}$ for $n<\omega$ and $\bigcap_{n<\omega} Y_{n}=\varnothing$. Further, the family $E \cup\left(Y_{n}\right)_{n<\omega}$ has the finite intersection property. Let $D$ be the smallest family of subsets of $S(\mathcal{C})$ containing $E,\left(Y_{n}\right)_{n<\omega}$, and closed under finite intersection.

The result of this section can be related to those of $\$ 2$ in the following way: Assume that $a=a^{a}>\omega$ and let $\mathcal{S}_{\alpha}$ be the $\alpha$-homogeneous-universal Boolean al. gebra of cardinality $\alpha$. We may regard $\delta_{\alpha}$ as a field of subsets of an appropriate subspace of $S\left(\delta_{\alpha}\right)$; nevertheless, no matter how this subspace is chosen, it is not possible to find a countably incomplete ultrafilter on $\delta_{a}$ (regarded as a field of sets of the subspace), since the intersection of less than $\alpha$ (and, in particular, of countably many) elements of $\mathcal{S}_{a}$ contains a nonzero element of $\mathcal{S}_{a}$ (by $1.4(\mathrm{~b})$ )). This can be rectified by the addition to $\mathcal{S}_{a}$ of the supremum of just one sequence $\left(V_{k}\right)_{k<\omega}$ of elements of $\delta_{a}$, where for simplicity we may assume that $V_{k} \neq \varnothing$ for $k<\omega$ and $V_{n} \cap V_{k}=\varnothing$ for $n<k<\omega$. By Lemma 4.2, it is clear that the smallest subalgebra $\mathcal{C}$ of the completion of $\mathcal{S}_{a}$ containing $\mathcal{S}_{\alpha}$ and the supremum of the family $\left(V_{k}\right)_{k<\omega}$ is a field of subsets of $S\left(\mathcal{S}_{\alpha}\right)$, such that the supremum of $\left(V_{k}\right)_{k<\omega}$ in $\mathcal{C}$ is equal to the set-theoretic union $\bigcup_{k<\omega} V_{k}$. But it is clear that $V_{k}, k<\omega$, is homeomorphic to $S\left(\mathcal{S}_{a}\right)$ (cf. Theorem 1.7 in $\left[\mathrm{N}_{1}\right]$ ). Thus $\mathcal{C}$ is isomorphic to the product $\mathcal{S}_{a}^{\omega} \times \mathfrak{T}$, where $\mathcal{I}$ is the Boolean algebra of open-and-closed subsets of $S\left(\mathcal{S}_{\alpha}\right) \backslash \bigcup_{k<\omega} V_{k}$. Since $S\left(\mathscr{S}_{\alpha}\right) \backslash \bigcup_{k<\omega} V_{k}$ is a compact space, there is a natural oneto-one correspondence between the countably incomplete ultrafilters of $\mathcal{C}$ (regarded, in the above sense, as a field of subsets of $S\left(\mathcal{E}_{a}\right)$ ), and the countably incomplete ultrafilters on $S_{a}^{\omega}$. (The same remarks are valid for any $\delta$, such that $\omega \leq \delta<\alpha$, in place of $\omega$.) Thus the simplest completion of $\mathcal{S}_{\alpha}$, which is necessary to produce a countably incomplete ultrafilter, leads to the results of $\$ 2$, and more generally of Part I. On the other hand, the $\alpha$-completion of $\mathcal{S}_{a}$ is the largest completion that does not increase the cardinality of $\mathcal{S}_{a}$, and which still is representable as an $\alpha$ complete field of subsets of $S\left(\mathfrak{d}_{a}\right)$.

These remarks give some informal insight in the relation of the results of $\$ 2$, of $\$ 4$ (of the present paper), and of Keisler's original results in $\left[K_{4}\right]$ : The methods of $\$ 2$ utilize essentially all the properties of the homogeneous-universal Boolean 
algebras (i.e., both $1.4(\mathrm{~b})$ and $1.4(\mathrm{c})$; cf. Theorem 1.7 in $\left[\mathrm{N}_{1}\right]$ to the effect that these properties are characteristic of $\delta_{a}$ ), which is natural since the fields of sets $\delta_{\alpha}^{\delta}, \omega \leq \delta<\alpha$, depart very slightly from $\delta_{\alpha}$, i.e., essentially just by the addition of a supremum for a "small" infinite family. The fields of sets $\mathfrak{S}_{a}^{(\alpha)}$ of $\$ 4$, on the other hand, must be regarded as an intermediate case between "homogeneity-universality" and "discreteness". There is a close enough relation between $\mathcal{S}_{a}$ in $\mathcal{S}_{a}^{(\alpha)}$ and the representability of $\mathcal{S}_{a}^{(\alpha)}$ as the $\alpha$-complete field of subsets of $S\left(\mathcal{S}_{\alpha}\right)$, a.generated by $\mathcal{S}_{a}$ to allow the derivation of the "disjoint refinement" lemma for $\mathcal{S}_{a}^{(a)}$ from the corresponding property of $\mathcal{S}_{a}$; but, everything else is dealt with in a discrete manner, i.e., in Keisler's original manner.

We note that between these two "extreme" cases there is a spectrum of similar results that can be established (notably, for the $\beta$-completions $\mathcal{S}_{a}^{(\beta)}$, $\omega<\beta \leq \alpha)$. For these cases, it is the lemma corresponding to 2.2 , or 4.4 , that will be the only nontrivial part of the proof. We will not go into any details.

An analysis of the foregoing arguments provides an extension (of the results of $\$ 4$ only) in a different direction: Pierce, in [P], studied the notion of an $\alpha$ atomic Boolean algebra, which is a generalization of the notion of an atomic Boolean algebra. Let us generalize further Pierce's concept as follows: Let $a$ be an infinite cardinal. A Boolean algebra $\mathcal{C}$ is called weakly $a$-atomic if $\mathcal{C}$ contains a dense set $D$ which is weakly a-compact in the following sense: (a) $\varnothing$ $\epsilon D$, (b) $D$ is closed under finite intersection, and (c) if $E \subset D^{*}(=D \backslash\{\varnothing\})$ is such that $|E|<\alpha$ and $E$ is closed under finite intersection, then there is $d \in D^{*}$, such that $d \subset e$ for all $e \in E$. By 1.4(a) and (b), an a-homogeneous-universal Boolean algebra $\mathcal{C}$ is atomless and weakly $a$-atomic, with $D=\mathcal{C}$. A class of examples of atomless, $\alpha^{+}$-atomic Boolean algebras is given in $\$ 2$ of [P].

Let $\mathcal{C}$ be an atomless, weakly $\alpha$-atomic Boolean algebra. Then the fundamental "disjoint refinement" lemma can be established by an imitation of the proof of 2.1. Also, assuming that $a$ is regular, the analogues of all the results of $\$ 4$, up to here, are established in identical fashion. Thus we obtain the following strengthening of Theorem $\mathrm{C}$.

Theorem $C^{\prime}$. Let $\alpha=\alpha^{a}>\omega$ and let $\mathcal{C}$ be an atomless, weakly $\alpha$-atomic Boolean algebra of cardinality $\alpha$. There is a countably incomplete, a-good ultrafilter on the a-completion $\mathcal{C}^{(\alpha)}$ (regarded as the a-complete field of subsets of $S(\mathcal{C})$ a.generated by $\mathcal{C})$.

5. Adequate ultrafilters on completions of special Boolean algebras. Recall that according to 1.1(d), $a=2^{\stackrel{N}{*}}$ if and only if either $a=a^{a}$ or $\alpha$ is a strong limit cardinal. We have already dealt with the case $a=\alpha^{a}$. We now consider the 
case that $\alpha$ is a strong limit cardinal. In contrast to the relation of $\$ 3$ to $\$ 2$, which has been one of generalization, the methods of $\$ 4$ apply only to cardinals $a$ of the form $a=a^{a}$ and the methods of $\$ 5$ only to strong limit cardinals. Lemmas 5.1 and 5.2 below are the (incomplete) analogues of 3.1 and 3.2 , respectively.

5.1. Lemma. Let $\beta$ be an infinite cardinal and set $\alpha=\left(2^{\beta}\right)^{+}$. We are given the following diagram

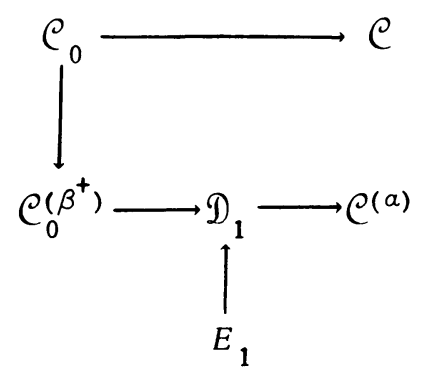

where

$\mathcal{C}_{0}$ is a $\beta^{+}$-homogeneous-universal Boolean algebra of cardinality $2^{\beta}$,

$\mathcal{C}$ is an a-bomogeneous-universal Boolean algebra of cardinality ${ }^{2}{ }^{\beta}$,

$\mathcal{C}_{0} \rightarrow \mathcal{C}$ is a Boolean algebra embedding (considered as an inclusion), and $\mathcal{C}_{0}^{(\beta)^{+}} \rightarrow \mathcal{C}^{(\alpha)}$ is the induced embedding (also considered as an inclusion),

$D_{1}$ is a $\beta^{+}$-complete Boolean algebra of cardinality $2^{\beta}$, sucb that $\mathcal{C}_{0}^{(\beta)^{+}} \mathrm{C}$ $\mathscr{D}_{1} \subset \mathcal{C}^{(\alpha)}$, and

$E_{1}$ is a subfamily of $\mathscr{D}_{1}^{*}$, closed under finite intersection.

Then there is a Boolean algebra $\mathfrak{T}_{2}$ (considered as a field of subsets of $s(\mathcal{C})$, such that

$\mathfrak{D}_{1} \subset \mathfrak{D}_{2} \subset \mathcal{C}^{(\alpha)}$, and

$\left|\mathfrak{D}_{2}\right|=2^{\beta}$, and

there is an ultrafilter $p_{2}$ on $\mathfrak{I}_{2}$, such that

$E_{1} \subset p_{2}$,

$p_{2}$ is countably incomplete, and

$p_{2}$ is $\left(\beta^{+}, E_{1}\right)$-good.

Proof. Note that $\left|E_{1}\right| \leq 2^{\beta}$, and hence that the set of (monotone) mappings from $S_{\omega}(\beta)$ to $E_{1}$ has cardinality $\left|E_{1}\right| S_{\omega}(\beta) \mid \leq\left(2^{\beta}\right)^{\beta}=2^{\beta}$. Let $\left\{\phi_{\eta}: \eta<2^{\beta}\right\}$ be a well-ordering of all these monotone mappings (with possible repetitions). We proceed by transfinite induction on $2^{\beta}$, using 4.4 at every stage (with $\alpha=\left(2^{\beta}\right)^{+}$).

First, using 4.5 , we adjoin to $E_{1}$ a countable family $\left(Y_{n}\right)_{n<\omega} \subset \bigodot^{(\alpha) *}$, such that $E_{1} \cup\left(Y_{n}\right)_{n<\omega}$ has the finite intersection property, and $\bigcap_{n<\omega}^{n<\omega} Y_{n}=\varnothing$. Let $D_{0}$ be the smallest family containing $E_{1} \cup\left(Y_{n}\right)_{n<\omega}$ and closed under finite intersection. 
For $0<\zeta<2^{\beta}, \zeta$ a limit ordinal, set $D_{\zeta}=\bigcup_{\eta<\zeta} D_{\eta}$.

We define $D_{\eta+1}$ for $\eta<2^{\beta}$. Consider $\phi_{\eta}: S_{\omega}(\beta) \rightarrow E_{1}$. We apply 4.4 to find a multiplicative mapping $\psi_{\eta}: S_{\omega}(\beta) \rightarrow \mathcal{C}^{(\alpha) *}$, such that

$\psi_{\eta} \leq \phi_{\eta}$, and

$\psi_{\eta}(F) \cap d \neq \varnothing$

for $F \in S_{\omega}(\beta), d \in D_{\eta}$. Let $D_{\eta+1}$ be the smallest subfamily of $\mathcal{C}^{(\alpha)}$, containing

$$
D_{\eta} \cup\left\{\psi_{\eta}(F) \cap d: F \in S_{\omega}(\beta), d \in D_{\eta}\right\},
$$

and closed under finite intersection. Inductively, it is easy to prove that $\left|D_{\eta}\right| \leq$ $2^{\beta}$ for all $\eta<2^{\beta}$, and that $D_{\eta}$ consists of elements of $\mathcal{C}^{(\alpha) *}$. Let $E_{2}=\bigcup_{\eta<2} D_{\eta^{*}}$. Thus $\left|E_{2}\right| \leq 2^{\beta}$. Let $\mathscr{D}_{2}$ be a Boolean subalgebra of $\mathcal{C}^{(\alpha)}$, containing $E_{2}$ and $\mathscr{D}_{1}$, and of cardinality $2^{\beta}$. Let $p_{2}$ be an ultrafilter on $\mathscr{D}_{2}$, containing $E_{2}$. Clearly, $p_{2}$ is $\left(\beta^{+}, E_{1}\right)_{\text {-good. }}$

\subsection{Lemma. Let the assumptions of this lemma be identical with those of}

5.1. Then, there is a Boolean algebra $\mathfrak{D}$ (considered as a field of subsets of $s(\mathcal{C})$, sucb that

$$
\begin{aligned}
& \mathscr{D}_{1} \subset \mathscr{D} \subset \mathcal{C}^{(a)}, \\
& |\mathfrak{D}|=2^{\beta}, \\
& \mathcal{D} \text { is } \beta^{+} \text {-complete, and }
\end{aligned}
$$

there is an ultrafilter $p$ on $\mathcal{D}$, such that

$E_{1} \subset p$,

$p$ is countably incomplete, and

$p$ is $\beta^{+}$-good.

Proof. We proceed by transfinite induction on $\beta^{+}$, using 5.1 at every stage. We start with $\mathscr{D}_{1}, E_{1}$.

Let $1<\xi<\beta^{+}$. Assume inductively, that for all $\zeta$, $1 \leq \zeta<\xi$, we have defined Boolean subalgebras $\mathfrak{D}_{\zeta}$ of $\mathcal{C}^{(\alpha)}$ and ultrafilters $p_{\zeta}$ on $\mathfrak{D}_{\zeta}$. We define $\mathfrak{D}_{\xi}, p_{\xi}$ :

If $\xi$ is a limit ordinal, set $\mathfrak{D}_{\xi}=\bigcup_{\zeta<\xi} \mathfrak{D}_{\zeta}$ and $p_{\xi}=\bigcup_{\zeta<\xi} p_{\zeta}$.

Let $\xi=\zeta+1$. We use 5.1 with $\mathscr{D}_{\zeta}, p_{\zeta}$ (in place of $\left.\mathfrak{D}_{1}, p_{1}\right)$. The lemma gives $D_{\zeta}, p_{\zeta}$ satisfying the conclusion of 5.1 , in the present context. It is easy to prove inductively that $\left|\mathscr{D}_{\xi}\right| \leq 2^{\beta}$ for $\xi<\beta^{+}$. Let $\mathscr{D}=\bigcup_{\xi<\beta^{+}} \mathfrak{D}_{\xi}$ and $p=\bigcup_{\xi<\beta^{+}} p_{\xi}$. The conclusions of the present lemma are clearly satisfied.

Incidentally, we have proved the following general embedding property.

5.3. Corollary. Let $\mathcal{C}$ be a Boolean algebra (regarded as a field of sets) such that $|\mathcal{C}| \leq 2^{\beta}$, and let $E \subset \mathcal{C}^{*}$, such that $E$ is closed under finite intersection.

Then, there is a field of sets $\mathcal{D}$, such that

$\mathcal{C} \subset \mathcal{D}$,

$|\mathcal{D}|=2^{\beta}$,

$D$ is $\beta^{+}$-complete, and 
there is an ultrafilter $p$ on $\mathcal{D}$, such that

$E \subset p$,

$p$ is countably incomplete, and

$p$ is $\beta^{+}$-good.

Proof. By 1.2, we may embed $\mathcal{C}$ into a $\beta^{+}$-homogeneous-universal Boolean algebra $\mathcal{C}_{1}$ of cardinality $2^{\beta}$, and, again by 1.2 , we may embed $\mathcal{C}_{1}$ into a $\left(2^{\beta}\right)^{+}$. homogeneous-universal Boolean algebra $\mathcal{C}_{2}$ of cardinality $2^{2}{ }^{\beta}$; the conclusion follows from an application of 5.2.

The following uniqueness property of certain completions of the special Boolean algebras can be proved without difficulty, by employing induction on all $\beta$ 's smaller than $\alpha$.

5.4. Lemma. Let $\alpha$ be an uncountable strong limit cardinal and let $\mathcal{S}_{a}$ be the special Boolean algebra of cardinality a. Let $\left\{\mathcal{C}_{\beta}, \beta\right.$ 's $\left.<\alpha\right\}$ and $\left\{\mathscr{D}_{\beta}, \beta<\alpha\right\}$ be two specializing chains for $\mathfrak{S}_{\alpha}$, sucb that $\left|\mathcal{C}_{\beta}\right|=\left|\mathscr{D}_{\beta}\right|=2^{\beta}$ for $\beta<\alpha$. Then there is an isomorphism

$$
\phi: \bigcup_{\beta<a} \mathcal{C}_{\beta}^{\left(\beta^{+}\right)} \cong \bigcup_{\beta<\alpha} \mathcal{D}_{\beta}^{\left(\beta^{+}\right)}
$$

such that $\phi \mid \mathcal{S}_{a}$ is the identity on $\mathcal{S}_{\alpha}$.

In view of this lemma, we denote by $\mathcal{S}_{\alpha}^{[\alpha]}$ the unique up to isomorphism union $\bigcup_{\beta<a} \mathcal{C}_{\beta}^{(\beta+)}$, where $\left\{\mathcal{C}_{\beta}: \beta<\alpha\right\}$ is a specializing chain of $\mathcal{S}_{a}$, such that $\left|\mathcal{C}_{\beta}\right|=$ $2^{\beta}$ for $\beta<\alpha$. Clearly, by $1.5,\left|\mathcal{S}_{\alpha}^{[a]}\right|=\alpha$.

We are now in position to prove the main result of this section.

Theorem D. Let $a$ be an uncountable strong limit cardinal, let $\delta_{a}$ denote the unique special Boolean algebra of cardinality $\alpha$, and let $\left\{\mathcal{C}_{\beta}, \beta<\alpha\right\}$ be a specializing chain for $\mathcal{S}_{\alpha}$, such that $\left|\mathcal{C}_{\beta}\right|=2^{\beta}$ for $\beta<a$. Then there is a chain $\left\{D_{\beta}, \beta<\alpha\right\}$ of subfields of sets of $\mathcal{S}_{\alpha}^{[a]}$, such that

$\left|\mathfrak{D}_{\beta}\right|=2^{\beta}$,

$D_{\beta}$ is a $\beta^{+}$-complete field of sets,

$\mathcal{C}_{\beta}^{\left(\beta^{+}\right)} \subset \mathscr{D}_{\beta} \subset \mathcal{C}_{2 \beta}^{\left(\left(2^{\beta}\right)^{+}\right)}$for $\beta<\alpha$, and

there is an adequate ultrafilter $p$ on $\mathcal{S}_{\alpha}^{[\alpha]}$, i.e., an ultrafilter that satisfies the following conditions:

$p_{\beta}=p \cap \mathscr{D}_{\beta}$ is countably incomplete, and

$p_{\beta}$ is $\beta^{+}$-good for $\beta<a$.

Proof. We employ transfinite induction on all cardinals less than $\alpha$, using 5.2 at every stage. Let $\beta<\alpha$. Assume inductively that for every cardinal $\gamma$, $\gamma<\beta$, we have defined fields of subsets of $\int_{a}^{[a]}, \mathfrak{D}_{\gamma}$, and ultrafilters $p_{\gamma}$ on $\mathfrak{D}_{\gamma}$, such that 
$\left|D_{\gamma}\right|=2^{\gamma}$,

$\mathfrak{D}_{\gamma}$ is a $\gamma^{+}$-complete field of sets,

$\mathcal{C}_{\gamma}^{\left(\gamma^{+}\right)} \subset \mathfrak{D}_{\gamma} \subset \mathfrak{D}_{2 \gamma}^{\left(\left(2^{\gamma}\right)^{+}\right)}$,

$\mathfrak{D}_{\gamma} \subset \mathfrak{D}_{\delta}$ for $\gamma<\delta<\beta$,

$p_{\gamma}=p_{\delta} \cap \mathscr{D}_{\gamma}$ for $\gamma<\delta<\beta$,

$p_{\gamma}$ is countably incomplete, and

$p_{\gamma}$ is $\gamma^{+}$-good.

We now define $\mathscr{D}_{\beta}, p_{\beta}$ as follows: Let $\widetilde{E}_{\beta}=U_{\gamma<\beta} \mathscr{D}_{\gamma}$ and $\widetilde{q}_{\beta}=U_{\gamma<\beta} p_{\gamma}$. Clearly, $\left|\mathscr{E}_{\beta}\right|=2^{\beta}$ and $\widetilde{E}_{\beta} \subset \mathcal{C}_{2}^{\left(\left(22^{+}\right)^{+}\right)}$. Let $\widetilde{D}_{\beta}$ be the $\beta^{+}$-complete subfield of $\mathcal{C}_{2 \beta}^{\left.\left(2^{\beta}\right)^{+}\right)}, \beta^{+}$-generated by $\widetilde{E}_{\beta} \cup \mathcal{C}_{\beta}^{\left(\beta^{+}\right)}$, and let $E_{\beta}=\widetilde{q}_{\beta}$. We employ 5.2 with $\widetilde{D}_{\beta}$, $E_{\beta}$, in place of $\mathscr{D}_{1}, E_{1}$ respectively, to obtain a subfield $\mathfrak{D}_{\beta}$ of $\left.\mathcal{C}_{2}{ }_{2}\left(2^{\beta}\right)^{+}\right)$, such that

$$
\begin{aligned}
& \widetilde{D}_{\beta} \subset \mathscr{D}_{\beta} \subset \mathcal{C}_{2 \beta}^{\left.\alpha\left(2^{\beta}\right)^{+}\right)}, \\
& \left|\mathscr{D}_{\beta}\right|=2^{\beta}, \\
& \mathscr{D}_{\beta} \text { is } \beta^{+} \text {-complete, and }
\end{aligned}
$$

an ultrafilter $p_{\beta}$ on $\mathfrak{D}_{\beta}$, such that

$E_{\beta} \subset p_{\beta}$,

$p_{\beta}$ is countably incomplete, and

$p_{\beta}$ is $\beta^{+}$-good.

This completes the inductive definition of $\mathfrak{D}_{\beta}, p_{\beta}$ for $\beta<\alpha$. Clearly, $\bigcup_{\beta<\alpha} \mathfrak{D}_{\beta}$ $=\mathfrak{S}_{a}^{[\alpha]}$. Let $p=\bigcup_{\beta<a} p_{\beta}$. It is easy to verify that $p$ is an adequate ultrafilter on $\mathcal{S}_{a}^{[a]}$.

\section{BIBLIOGRAPHY}

[B] Heinz Bachmann, Transfinite Zahlen, Zweite Auflage, Ergebnisse der Mathematik und ihrer Grenzgebiete, Band 1, Springer-Verlag, Berlin and New York, 1967. MR 36 \#2506.

[BS] J. L. Bell and A. B. Slomson, Models and ultraproducts: An introduction, NorthHolland, Amsterdam, 1969. MR 42 \#4381.

[CK] C. C. Chang and H. Jerome Keisler, Continuous model theory, Ann. of Math. Studies, no. 58, Princeton Univ. Press, Princeton, N. J., 1966. MR 38 \#36.

[EGH] P. Erdös, L. Gillman and M. Henriksen, An isomorphism theorem for real-closed fields, Ann. of Math. (2) 61 (1955), 542-554. MR 16, 993.

[ES] S. Eilenberg and N. E. Steenrod, Foundations of algebraic topology, Princeton Univ. Press, Princeton, N. J., 1952. MR 14, 398.

[G] Fred Galvin, Reduced products, Horn sentences, and decision problems, Bull. Amer. Math. Soc. 73 (1967), 59-64. MR 35 \#39.

[H1 $\mathrm{H}_{1}$. Hausdorff, Der Potenzbegriff in der Mengenlehre, Jber. Deutsch. Math. Verein. 13 (1904), 569-571. 435-505.

$\left[\mathrm{H}_{2}\right]-$ Grundzuge einer Theorie der geordneten Mengen, Math. Ann. 65 (1908),

[ $\left.\mathrm{J}_{1}\right]$ B. Jónsson, Universal relational systems, Math. Scand. 4 (1956), 193-208. MR $20 \# 3091$.

[J 2 ] Homogeneous universal relational systems, Math. Scand. 8 (1960), 137142. MR 23 \#A2328. 
[JO] B. Jónsson and P. Olin, Almost direct products and saturation, Compositio Math. 20 (1968), 125-132. MR 37 \#2589.

[K $\left.\mathrm{K}_{1}\right]$ H. Jerome Keisler, A characterization of Horn classes, Notices Amer. Math. Soc. 6 (1959), 521. Abstract \#559-139.

[ $\left.\mathrm{K}_{2}\right] \longrightarrow$ - Ultraproducts and elementary classes, Nederl. Akad. Wetensch. Proc. Ser. A 64 = Indag. Math. 23 (1961), 477-495. MR 25 \#3816.

[K $\mathrm{K}_{3}$ H. Jerome Keisler, Limit ultrapowers, Trans. Amer. Math. Soc. 107 (1963), 382408. MR $26 \# 6054$.

[K4] - Good ideals in fields of sets, Ann. of Math. (2) 79 (1964), 338-359. MR $29 \# 3383$.

[K5] - Ultraproducts and saturated models, Nederl. Akad. Wetensch. Proc. Ser. A 67 = Indag. Math. 26 (1964), 178-186. MR 29 \#5745.

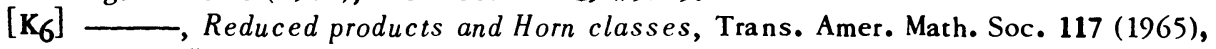
307-328. MR $30 \# 1047$.

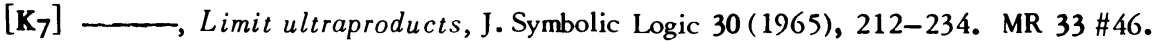

[Ko] Simon Kochen, Ultraproducts in the theory of models, Ann. of Math。 (2) 74 (1961), 221-261. MR $25 \# 1992$.

[Ku] K. Kunen, Ultrafilters and independent sets (manuscript)。

[Kur] C. Kuratowski, Sur l'extension de deux théorèmes topologiques à la théorie des ensembles, Fund. Math. 34 (1947), 34-38. MR 8, 505. $297-323$.

[M1] R. Mansfield, The theory of Boolean ultropowers, Ann. Math. Logic 2(1971),

$\left[\mathrm{M}_{2}\right]-$, Horn classes and reduced direct products (manuscript).

[MV] M. Morley and R. Vaught, Homogeneous universal models, Math. Scand. 11 (1962), 37-57. MR 27 \#37.

[ $\left.\mathrm{N}_{1}\right]$ S. Negrepontis, The Stone space of the saturated Boolean algebras, Trans. Amer. Math. Soc. 141 (1969), 515-527. MR 40 \#1311.

$\left[\mathrm{N}_{2}\right]-$, A property of the saturated Boolean algebras, Nederl. Akad. Wetensch. Proc. Ser. A 74 = Indag. Math. 33 (1971), 117-120.

$\left[\mathrm{N}_{3}\right]-$ Adequate ultrafilters of special Boolean algebras, Notices Amer. Math. Soc. 17 (1970), 832-833. Abstract \#70T-E53.

[P] R. S. Pierce, A generalization of atomic Boolean algebras, Pacific J. Math. 9 (1959), 175-182. MR 21 \#5591.

[Sh] S. Shelah, Every two elementarily equivalent models have isomorphic ultrapowers, Israel J. Math. 10 (1971), 224-234.

[s] Roman Sikorski, Boolean algebras, 2nd ed., Ergebnisse der Mathematik und ihrer Grenzgebiete, N. F., Band 25, Academic Press, New York; Springer-Verlag, Berlin, 1964. MR $31 \# 2178$.

[T] Alfred Tarski, Quelques théorèmes sur les alephs, Fund. Math. 7 (1925), 1-14.

DEPARTMENT OF MATHEMATICS, McGILL UNIVERSITY, MONTREAL 101, QUEBEC, CANADA

Current address: Department of Mathematics, Athens University, Athens 621, Greece 